\title{
Right Angle Free Subsets in the Plane
}

\author{
B. Gamble ${ }^{1}$, W. Pulleyblank ${ }^{2}$, B. Reed ${ }^{3}$ and B. Shepherd ${ }^{4}$ \\ 1 Kellogg School of Management, Northwestern University, Chicago, IL, USA \\ 2 T.J. Watson Research Center, IBM, Yorktown Heights, NY, USA. \\ 3 Department of Combinatorics and Optimization, University of Waterloo, Waterloo, \\ Ontario, Canada.* \\ 4 Centre for Mathematics and Computer Science, P.O. Box 4079, 1009AB Amsterdam, \\ The Netherlands.
}

\begin{abstract}
In this paper we investigate the complexity of finding maximum right angle free subsets of a given set of points in the plane. For a set of rational points $P$ in the plane, the right angle number $\rho(P)$ (respectively rectilinear right angle number $\rho_{R}(P)$ ) of $P$ is the cardinality of a maximum subset of $P$, no three members of which form a right angle triangle (respectively a right angle triangle with its side or base parallel to the $x$-axis). It is shown that both parameters are $\mathcal{N P}$-hard to compute. The latter problem is also shown to be equivalent to finding a minimum dominating set in a bipartite graph. This is used to show that there is a polynomial algorithm for computing $\rho_{R}(P)$ when $P$ is a horizontally-convex subset of the lattice $\mathbb{Z} \times \mathbb{Z}(P$ is horizontally-convex if for any pair of points in $P$ which lie on a horizontal line, every lattice point between them is also in $P$ ). We then show that this algorithm yields a $\frac{1}{2}$-approximate algorithm for the right angle number of a convex subregion of the lattice.
\end{abstract}

\section{Introduction}

Let $P$ be a set of points in the plane. A subset $S$ of $P$ is right angle free (raf) if no three points of $S$ form the vertices of a right angle triangle. The right angle number of $P$, denoted by $\rho(P)$, is the cardinality of a maximum raf subset of $P$.

Let $f(n)$ be the largest integer such that for any set $P$ of $n$ points in the plane, $\rho(P) \geq f(n)$. Erdös and Silverman [5] noted that a $k \times k$ rectangular subset of the integer lattice has right angle number $2 k-2$. Hence $f\left(k^{2}\right) \leq 2 k-2$. Abbott [1] proved that $f(n) \geq \sqrt{n}$. It is still unknown, however, whether $\lim _{n \rightarrow \infty} f(n) / n^{1 / 2}$ exists.

For points $x=\left(x_{1}, x_{2}\right), y=\left(y_{1}, y_{2}\right)$ the taxi-cab distance between $x$ and $y$ is $\left|x_{1}-y_{1}\right|+\left|x_{2}-y_{2}\right|$. The $k$-diamond, $D_{k}$, is the set of all points on the integer lattice which are within taxi-cab distance $k$ of the origin. $\left(D_{3}\right.$ is shown in Fig. 1). The set of points on either axis forms an raf subset of $D_{k}$ of size $2 k+1$, and

* Current address: Departments of Mathematics and Operational Research, London School of Economics, Houghton Street, London WC2A 2AE. 


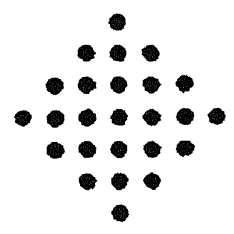

Fig. 1

Abbott conjectured that these were of maximum possible size. However, Paul Lemke [6] showed that raf subsets of size greater than $2 k+1$ exist in $D_{k}$, for some vales of $k$. However, as $k \rightarrow \infty$, the ratio of the size of these sets to $2 k+1$ converges to 1 . He asks whether in fact $\lim _{k \rightarrow \infty} \rho\left(D_{k}\right) /(2 k+1)=1$. This would imply that if $f(n)$ exists, $\lim _{n \rightarrow \infty} f(n) / n^{1 / 2} \leq \sqrt{2}$.

In this paper we examine an associated computational decision problem, which we call RAF: Given an integer $k$ and finite set $\boldsymbol{P}$ of rational points in the plane, determine whether $\rho(P) \geq k$.

We also consider a related problem where we only prohibit right angle triangles having a prescribed set of orientations. A set of points $S$ in the plane is rectilinear right angle free (rraf) if no three points of $S$ form a right angle triangle with a base side which is parallel to the vector $(1,0)$, i.e., a base which is horizontal. For a set $P$ of points, the rectilinear right angle number of $P$, denoted by $\rho_{R}(P)$, is the cardinality of a maximum rraf subset of $P$. (Note that $\rho_{R}(P) \geq \rho(P)$.) We let RRAF denote the decision problem: Given an integer $k$ and set of points $P$, determine whether $\rho_{R}(P) \geq k$.

An algorithm to solve RAF is polynomial if its running time is bounded by a polynomial in the number of binary bits needed to encode the given set of points as input. A pseudo-polynomial algorithm is defined similarly but the points in the plane may be encoded in unary. A problem in $\mathscr{N P}$ is strongly $\mathscr{N} \mathscr{P}$-complete if the existence of a pseudo-polynomial algorithm for that problem implies the existence of a polynomial algorithm for some $\mathscr{N} \mathscr{P}$-complete problem.

In the next section we show that both RAF and RRAF are strongly $\mathscr{N P}$ complete. In Section 3 we show that RRAF is polynomially solvable for sets $P$ satisfying a certain convexity condition. This is used to give a 1/2-approximate algorithm for RAF restricted to convex subregions of the lattice.

\section{Complexity Results}

\section{$2.1 R A F$}

Clearly RAF is in $\mathcal{N P}$, for we can verify that a set $P$ is right angle free by considering each triple of points in turn and ensuring that no two of the lines defined by the pairs of points of the triple are orthogonal. We show that RAF is strongly $\mathscr{N} \mathscr{P}$-complete by showing that existence of a pseudo-polynomial algorithm for RAF implies the existence of a polynomial algorithm to solve the stable set prob- 
lem for a general graph. As usual, $\alpha(G)$ denotes the size of a largest stable set in $G$. We begin with a few definitions.

We say $z$ centres a right angle with endpoints $x$ and $y$ if $(x, z, y)$ forms a right angle triangle with hypotenuse $\overline{x y}$. For points $x$ and $y$, denote by $C(\{x, y\})$, the circle whose diameter is determined by $\overline{x y}$. Then $z$ centres a right angle with endpoints $x, y$ if and only if $z \in C(\{x, y\})$. Thus a set of points $S$, is raf if and only if $C(\{x, y\}) \cap S=\{x, y\}$ for all $\{x, y\} \subseteq S$.

Theorem 2.1. $R A F$ is strongly $\mathscr{N P}$-complete.

Proof. Let $G=(\{1, \ldots, n\}, E)$ be a graph. We create an instance $P$ of RAF of size $n+|E|$ for which $\alpha(G)=|E|-\rho(P)$. Set $V^{\prime}=\{\bar{i}=(i, 0): 1 \leq i \leq n\}$. Construct a set $E^{\prime}$ of points iteratively by initializing $E^{\prime}=\varnothing$ and then repeating the following for each edge $i j$, in turn:

- Pick a point $z$ in the first quadrant of $C(\{\bar{i}, \bar{j}\})$, that is, belonging to $C^{\mathbf{1}}(\{\dot{i}, \bar{j}\}) \equiv$ $\left\{\left(x_{1}, x_{2}\right) \in C(\{\dot{i}, \bar{j}\}): x_{2}>0, x_{1}>(j+i) / 2\right\}$, such that:

$\left({ }^{*}\right) z$ is only in the right triangle $(\bar{i}, z, \bar{j})$.

We call $\bar{i}, \bar{j}$ the endpoints of $z$.

- Set $E^{\prime}=E^{\prime} \cup\{z\}$.

Let $P=V^{\prime} \cup E^{\prime}$.

Claim. $\rho(P)=|E|+\alpha(G)$.

Proof. By construction each $z$ in $P$ is in exactly one right angle triangle of $P$-the one associated with its endpoints (i.e. an edge of $G$ ). Thus for any independent subset $I$ of $G, E^{\prime} \cup\{\bar{i}: i \in I\}$ is raf. Hence $\rho(P) \geq|E|+\alpha(G)$.

Now let $S$ be a maximum raf subset of $P$ which maximizes $\left|S \cap E^{\prime}\right|$. If there exists $z \in E^{\prime}-S$, then by maximality both endpoints of $z$, say $x$ and $y$ are in $S$. But then $(S-\{x\}) \cup\{z\}$ is raf, a contradiction. Hence $E^{\prime} \subseteq S$ and so for any $\bar{i}$, $\bar{j} \in S \cap V^{\prime}$ we must have $i j \notin E$. Thus $|S| \leq\left|E^{\prime}\right|+\alpha(G)=|E|+\alpha(G)$.

Some details still remain. In particular, how do we construct $E^{\prime}$ in time polynomially bounded in $n$ ? It suffices to show that the following problems can be solved in polynomial time:

(a) Determining whether $x$ and $y$ are endpoints of a right angle with centre $z$;

(b) Augmenting $E^{\prime}$.

We first note that if $x, y$ and $z$ are rational points, then the problem (a) can be answered by evaluating an equation of the form

(1) $\left(z_{1}-\left(\left(y_{1}+x_{1}\right) / 2\right)\right)^{2}+\left(z_{2}-\left(\left(y_{2}+x_{2}\right) / 2\right)\right)^{2}=\left(\left(x_{1}-y_{1}\right)^{2}+\left(y_{2}-x_{2}\right)^{2}\right) / 4$

Now consider problem (b), finding a point $z$ satisfying $\left({ }^{*}\right)$. First we show that there is a polynomial $p(\cdot)$ such that at most $p(n)$ points on $C^{1}(\{\bar{i}, \bar{j}\})$ fail condition $\left.{ }^{*}\right)$. Consider any candidate $z^{(1)}$, for augmenting $E_{1}^{\prime}$. Let the endpoints of $z^{(1)}$ be $\bar{i}$ and $\bar{j}$, i.e., $z^{(1)} \in C^{1}(\{\bar{i}, \bar{j}\}) \cap Q \times Q$. The condition $\left(^{*}\right)$ is satisfied by $z^{(1)}$ if

$$
z \notin C(\{u, v\}) \quad \text { for all }\{u, v\} \subseteq\left(E^{\prime} \cup V^{\prime}\right)-\{\bar{i}, \bar{j}\}
$$


and

$$
\left|C(\{z, \bar{i}\}) \cap\left(V^{\prime} \cup E^{\prime}\right)\right|=\left|C(\{z, \bar{j}\}) \cap\left(V^{\prime} \cup E^{\prime}\right)\right|=1
$$

(To check whether (2) and (3) hold requires at most $\left(\begin{array}{c}\left|V^{\prime} \cup E^{\prime}\right| \\ 2\end{array}\right)$ evaluations of the form given in (1).) If one of the conditions fails, then pick another candidate $z^{(2)} \in C^{1}(\{\bar{i}, \bar{j}\})-z^{(1)}$. Since any circle (line) intersects quadrant one of $C(\{\bar{i}, \bar{j}\})-$ $\{\bar{j}\}$ in at most one point we know that condition (2) (respectively (3)) can fail for at most $\left(\begin{array}{c}\left|V^{\prime} \cup E^{\prime}\right|-2 \\ 2\end{array}\right)$ (respectively $2\left|V^{\prime} \cup E^{\prime}\right|-2$ ) distinct points. Thus after at $\operatorname{most}\left(\begin{array}{c}\left|V^{\prime} \cup E^{\prime}\right| \\ 2\end{array}\right)=p(n)$ candidates we are able to augment $E^{\prime}$.

Now we know that we need only generate a polynomial number of points on a circle for each new edge considered. We must show how to find these points and how to make sure that their sizes do not grow too quickly. Given any Pythagorean Triple $(a, b, c)$, where we assume $a \geq b$, the point

$$
z=\left(j-(j-i) b^{2} / c^{2},(j-i) a b / c^{2}\right)
$$

is rational and lies on $C(\{\bar{i}, \bar{j}\})$. Since the equations

$$
a=2 m(m+1), \quad b=(m+1)^{2}-m^{2}, \quad c=m^{2}+(m+1)^{2}
$$

yield distinct Pythagorean Triples, we can always generate a $z$ with unary representation bounded by a polynomial in $n$.

This completes the proof of Theorem 2.1 .

A set $P$ of points in $\mathbb{Z} \times \mathbb{Z}$ is horizontally-convex if for any two points of $P$ which lie on a horizontal line, each lattice point between them is also in $P$. We define vertically-convex in a similar fashion and say that the subset $P$ is rectilinearconvex if it is both horizontally and vertically-convex. We can modify the reduction in Theorem 2.1 so that each member in $P$ is alone in its row and column. This is done by "tilting" the configuration slightly. We can also scale the resulting set of points so that it lies on the integer lattice. This shows that RAF remains $\mathscr{N} \mathscr{P}$-hard for rectilinear-convex regions.

\section{$2.2 R R A F$}

For $G=(V, E)$, a subset $D$ of $V$ is a dominating set of $G$ if each vertex of $V-D$ is adjacent to some vertex of $D$. The domination number of $G$, denoted by $\gamma(G)$, is the size of a smallest dominating set of $G$. We show that RRAF is $\mathscr{N} \mathscr{P}$-complete, by showing that this problem is equivalent to the problem of finding minimum dominating sets in bipartite graphs, which is $\mathscr{N} \mathscr{P}$-complete (Bertossi [2], Chang and Nemhauser [3]).

We start by defining a bipartite graph $B(P)$, associated with a set of points $P$ in the plane. The graph $B$ has bipartition $(H(P), V(P))$. Roughly speaking, the members of $H(P)$ correspond to the horizontal lines in the plane which contain points of $P$. Similarly $V(P)$ corresponds to the vertical lines which contain points 
of $P$. More precisely, we let $H(P)=\left\{b_{y_{0}}\right.$ : there exists $x$ such that $\left.\left(x, y_{0}\right) \in P\right\}$, and $V(P)=\left\{a_{x_{0}}\right.$ : there exists $y$ such that $\left.\left(x_{0}, y\right) \in P\right\}$ (where $a$ and $b$ are indeterminates). Also $E(P)=\left\{a_{x} b_{y}:(x, y) \in P\right\}$ and $B(P)=(H(P) \cup V(P), E(P))$. Clearly $B(P)$ is a bipartite graph. The next theorem shows how $\gamma(B)$ is related to $\rho_{R}(P)$.

Theorem 2.2. For any set of points $P, \rho_{R}(P)+\gamma(B(P))=|H(P) \cup V(P)|$.

Proof. A subset $S$ of $P$, is rraf if and only if the subset of $E(P)$ corresponding to $S$ induces a subgraph of $B(P)$ without a path of length three. It is easy to see there exists a maximum such subset $E^{\prime}$ of $E(P)$ for which $B\left[E^{\prime}\right]$ (the graph induced by the edges $E^{\prime}$ ) has no isolated vertices. Hence if we take from each component of $B\left[E^{\prime}\right]$, a vertex of maximum degree we obtain a dominating set for the bipartite graph $B(P)$. Thus $\gamma(B(P)) \leq|H \cup V|-\rho_{R}(P)$. Conversely let $D$ be a minimum dominating set of $B(P)$ and consider a minimal subset $E^{\prime}$ of $E(P)$ such that $D$ is a dominating set of $B\left[E^{\prime}\right]$. Evidently $R\left[E^{\prime}\right]$ has no path of length 3 , and so $\rho_{R}(P) \geq$ $|H \cup V|-\gamma(B(P))$. Hence $\rho_{R}(P)+\gamma(B)=|H \cup V|$.

Corollary 2.3. $R R A F$ is strongly $\mathscr{N P P}$-complete.

Proof. It is immediate that a pseudo-polynomial algorithm for RRAF implies the existence of a polynomial algorithm to solve the domination problem for bipartite graphs. Since this problem is $\mathscr{N} \mathscr{P}$-complete, the result follows.

By defining a certain type of weighted RAF problem, we obtain a problem equivalent to the minimum weight domination problem. Given weights on the $x$ and $y$ coordinates, the induced weight of an rraf subset $S$ corresponds to summing the weights of the vertices in $S$. However, these weights depend on $S$ as follows. If $(x, y) \in S$ is not alone in its row, then it assumes the weight of its $y$ coordinate. Similarly, if there is another vertex in $S$ with same $x$ coordinate, then it assumes the weight of its $x$ coordinate. If $(x, y)$ is alone in its row and column, then its weight becomes the maximum of the corresponding coordinate weights. The induced weight RRAF problem is find a maximum induced weight rraf subset in a given set of points. We leave it as an exercise to show that Theorem 2.2 can be extended to give a similar relation between weighted dominating set problem and the induced weight RRAF problem.

\section{RRAF in Horizontally Convex Sets}

We now show that there is a polynomial time algorithm to solve RRAF if the input consists of a horizontally-convex subset of the lattice. In fact if $P$ is horizontally convex, then in $B(P)$ each vertex of $H(P)$ is adjacent to a "contiguous" block of vertices in $V(P)$. Such a bipartite graph, for which the vertices in one part of the bipartition can be ordered so that each vertex in the other part is adjacent to a contiguous block of vertices, is called convex. We give an extension of the cardinality algorithm outlined in [4] which finds a minimum weight dominating set in a convex graph in polynomial time. 
Theorem 3.1. The weighted domination problem for convex graphs can be solved in polynomial time.

Corollary 3.2. The problem $R R A F$ restricted to horizontally-convex sets is solvable in polynomial time.

We present an algorithm for finding a minimum weight dominating set in a convex graph $B=(V=(I \cup J), E)$ which has positive weights $w \in \mathbb{Z}^{V}$ assigned to its vertices. Assume that $J$ is ordered in such a way that the neighbourhood of each vertex in $I$ forms a contiguous block of vertices. Let $J_{1}, J_{2}, \ldots J_{m}$ be a partition of $J$ such that each $J_{i}$ is a maximal contiguous subset of $J$, all of whose members have identical neighbourhoods (in $n$ ). We let $I_{i}=N\left(J_{i}\right)$ and let $\mathscr{T}(i)=$ $J_{1} \cup \cdots \cup J_{i}$, for $i=1,2, \ldots m$. Let $F_{i}=\{x \in I: N(x) \subseteq \mathscr{T}(i)\}$. Note, for any dominating set $D$ of $B$, each vertex in $F_{i}$ is either in $D$ or a neighbour in $\mathscr{T}(i)$ must be in $D$. For $z \in I$, we denote by $r(z)$ (respectively $l(z)$ ) the largest (respectively smallest) integer $i$ such that $z$ is adjacent to $J_{i}$. The general approach of our algorithm is to solve the problem inductively for $i=1, \ldots, m$. if

For any $k, t_{k} \in\{1, \ldots, m\}$, with $t_{k} \leq k$, we say that a set $D \subseteq V$ is of type $\left(k, t_{k}\right)$

$$
D \subseteq I \cup \mathscr{T}(k),
$$

$$
D \text { dominates all vertices in } \mathscr{T}\left(t_{k}\right) \text {, }
$$

$$
\text { and } D \text { dominates all vertices in } F_{k} \text {. }
$$

In general, this is a partial dominating set, which is contained in $I \cup \mathscr{T}(k)$, and which dominates all vertices in $\mathscr{T}\left(t_{k}\right)$ as well as any vertices in $I$ which can only be dominated by themselves or vertices in $\mathscr{T}(k)$.

It can be checked that any minimum weight dominating set will contain either 0,1 or all vertices of each $J_{k}$. For $c=0,1,2$ and $s=0, \ldots, k$, a set of type $\left(k, t_{k}\right)$ is said to satisfy $(c, s)$ if

$$
\begin{gathered}
J_{i} \cap D=\varnothing \text { for } i>s, \\
\left|J_{s} \cap D\right|=1 \text { if } c=1 \text { and } s>0, \\
\left|J_{s} \cap D\right|=0 \text { if } c=0 \text { or } s=0, \\
J_{s} \subseteq D \text { if } c=2 \text { and } s>0 .
\end{gathered}
$$

Basically, this means that $D$ contains no vertices of $J_{i}$ for $i>s$, and if $s>0$, then contains 0,1 or all vertices of $J_{s}$, depending on whether $c=0,1$ or 2 .

We construct a collection of sets $D_{k}\left(c, s, t_{k}\right)$, for $k=1, \ldots m$, for $c=0,1,2$, for $s=0, \ldots k$ and $t_{k} \leq k$ such that:

$$
\begin{aligned}
& D_{k}\left(c, s, t_{k}\right) \text { is a minimum weight }\left(k, t_{k}\right) \text { set satisfying }(c, s) ; \\
& \text { subject to minimality in (11), } D_{k}\left(c, s, t_{k}\right) \text { dominates } \mathscr{T}(i) \\
& \text { for maximum } i \geq t_{k} \text {. }
\end{aligned}
$$

Note that from such a collection we implicitly know a collection of sets $D_{k}\left(t_{k}\right)$ which are the minimum weight sets of type $\left(k, t_{k}\right)$ which also satisfy (12). 
We now show how to construct this collection in polynomial time. In the following we let $z_{i}$ be any minimum weight vertex of $I_{i}$, for which $r\left(z_{i}\right)$ is maximum.

The initial sets are obtained as follows: $D_{1}(2,1,0)=D_{1}(2,1,1)=J_{1}$; $D_{1}(1,1,0)=\{z\}$ for any $z \in J_{1}$ of minimum weight; $D_{1}(1,1,1)=D_{1}(1,1,0) \cup\left\{z_{1}\right\}$ $D_{1}(0,0,0)=F_{1}$ and finally

$$
D_{1}(0,0,1)= \begin{cases}F_{1} & \text { if } F_{1} \neq \varnothing \\ \left\{z_{1}\right\} & \text { otherwise. }\end{cases}
$$

We proceed to show how to construct a set $D_{k+1}\left(c, s, t_{k+1}\right)$ for $k \geq 1$, for $c=0,1$ or 2 , for $s=0, \ldots, k$.

Case $1 . k+1>s$.

First let $F$ be the subset of $F_{k+1}$ consisting of vertices adjacent to $J_{k+1}$ but not to $J_{s}$. If $F \neq \varnothing$, then let $j$ be the smallest integer such that some vertex of $F$ is adjacent to $J_{j+1}$. We then have:

(I) $D_{k+1}\left(c, s, t_{k+1}\right)=D_{l}(c, s, l) \cup F$

where $l=\min \left\{j, t_{k+1}\right\}$. When $F=\varnothing$, if either $D_{k}\left(c, s, t_{k+1}\right)$ dominates $J_{k+1}$ or $t_{k+1} \leq k$, we have:

(II) $D_{k+1}\left(c, s, t_{k+1}\right)=D_{k}\left(c, s, t_{k+1}\right)$.

Otherwise $t_{k+1}=k+1$ and $D_{k}(c, s, k)$ does not dominate $J_{k+1}$ and $D_{k+1}\left(c, s, t_{k+1}\right)$ becomes an appropriate minimum weight set in:

(III) $\left\{D_{k}(c, s, j) \cup\{z\}: z \in I_{k+1}\right.$ is a minimum weight vertex with $l(z)=j+1$ and subject to this maximizes $r(z)\}$.

It is easy to see that in Cases (I) and (II), the sets satisfy (11) and (12). Now consider the third case and let $D$ be any set satisfying (11) and (12) and let $z \in D \cap I_{k+1}$. It follows that $D-\{z\}$ is a type $(k, l(z)-1)$ set satisfying $(c, s)$. Hence there is a set of at most the weight of $D$ which is considered in (III). Since each of the sets in (III) is feasible, we easily deduce that we can find a new set satisfying (11) and so in fact also (12).

Case 2. $s=k+1, c=1$.

If $D_{k}\left(t_{k+1}\right)$ dominates $J_{k+1}$ or if $t_{k+1} \leq k$, then we have:

(IV) $D_{k+1}\left(1, s, t_{k+1}\right)=D_{k}\left(t_{k+1}\right) \cup\{z\}$

for any $z \in J_{k+1}$ of minimum weight. So suppose $t_{k+1}=k+1$ and $D_{k}\left(t_{k+1}\right)$ does not dominate $J_{k+1}$. We can argue as in (III) to show that a suitable set for our collection can be found in:

(V) $\left\{D_{k}(c, s, t) \cup\{z\} \cup\left\{z^{\prime}\right\}\right.$ : for all minimum weight $z^{\prime} \in J_{k+1}$ and $z \in I_{k+1}$ with $l(z)=t+1$ and all possible choices of $c, s\}$.

Case 3. $s=k+1, c=2$.

In this case it is easy to see that

$$
D_{k+1}\left(2, k+1, t_{k+1}\right)=D_{k}\left(\min \left\{k, t_{k+1}\right\}\right) \cup J_{k+1}
$$

gives a suitable set. 
The procedure described above can be performed in polynomial time. Hence we can find a minimum weight dominating set in a convex bipartite graph in polynomial time and so we can also compute $\rho_{R}$ for horizontally-convex sets.

In general, an algorithm which computes $\rho_{R}$ does not give a constant bound for the parameter $\rho$.

Theorem 3.3. There does not exist a positive constant $C$ such that $\rho(P) \geq C \rho_{R}(P)$ for every rectilinear-convex subset $P$ of $\mathbb{Z} \times \mathbb{Z}$.

Proof. For any nonnegative integer $n$ define $S_{n}$ as follows. Let $L_{n}$ consist of points $\{(i, i): i=1, \ldots, n\}$; define $L_{n}^{k}=\left\{x+(k,-k): x \in L_{n}\right\}$. Set $S_{n}=\bigcup_{k=0}^{n-1} L_{n}^{k n}$. Each $S_{n}$ is rectilinear-convex, indeed each point is alone in its row and column. Hence $\rho_{R}\left(S_{n}\right)=\left|S_{n}\right|=n^{2}$, but $S_{n}$ is just a "tilted" $n \times n$ grid and so $\rho\left(S_{n}\right)=2 n-2$.

If $P$ is forced to be convex however, such a bound for $\rho(P)$ does exist. (A subset of the integer lattice is convex if it is the set of integer points contained in some convex polygon.)

Theorem 3.4. For any convex subset $P$ of $\mathbb{Z} \times \mathbb{Z}, \rho(P) \geq(1 / 2) \rho_{R}(P)$.

Proof. Let $S$ be a maximum rraf subset of $P$. Define $S_{h}$ to be the points of $S$ for which there is another vertex of $S$ with the same $y$ coordinate. Similarly define $S_{v}$ to be the points of $S$ for which there is another vertex of $S$ with the same $x$ coordinate. Without loss of generality $\left|S_{h}\right| \geq\left|S_{v}\right|$. Set $S^{\prime}=S-S_{v}$. Note that no vertical line contains more than one point of $S^{\prime}$. Order the $y$ coordinates of the points in order of increasing $x$ coordinate, say $y_{1}, y_{2}, \ldots, y_{k}$ and suppose $S$ has been chosen to maximize the length of the initial monotone subsequence of $\left\{y_{i}\right\}_{i=1}^{k}$. We claim that $\left\{y_{i}\right\}_{i=1}^{k}$ itself is monotone. If not, then without loss of generality there is some $i$ such that $y_{1} \leq y_{2} \cdots \leq y_{i}, y_{l+1}<y_{i}$ and either $y_{i-1} \leq y_{i+1}$ or $y_{i+1} \leq y_{i-1}$. We consider only the case where $y_{i+1} \geq y_{i-1}$, the other case being similar.

For each $i$, let $x_{i}$ be the $x$ coordinate of the $i^{\text {th }}$ point in $S^{t}$. By convexity, every lattice point contained in the triangle determined by $\left(x_{i-1}, y_{i-1}\right),\left(x_{i}, y_{i}\right)$ and $\left(x_{i+1}, y_{i+1}\right)$ is in $P$. In particular $\left(x_{i}, y_{i+1}\right) \in P$. Thus $\left(S-\left\{\left(x_{i}, y_{i}\right)\right\}\right) \cup\left\{\left(x_{i}, y_{i+1}\right)\right\}$ contradicts the choice of $S$. Hence $\left\{y_{i}\right\}_{i=1}^{k}$ is monotone and so $S^{\prime}$ is raf and the theorem follows.

Corollary 3.5. There is a $\frac{1}{2}$-approximate algorithm for $R A F$ restricted to convex regions of the integer lattice.

Proof. Given $S$ a maximum rraf subset, we can polynomially construct $S^{\prime}$ as in Theorem 3.4.

Acknowledgements. We are grateful to Lorna Stewart for bringing reference [4] to our attention. The first and last authors acknowledge the support of post-graduate scholarships from the Natural Sciences and Engineering Research Council of Canada. The research of the second and third authors was partially supported by operating grants of the Natural Sciences and Engineering Research Council of Canada. 


\section{References}

1. Abbott, H.L.: On a Conjecture of Erdös and Silverman in Combinatorial Geometry. J. Comb. Theory, Ser. A 29, 380-381 (1980)

2. Bertossi, A.A.: Dominating sets for split and bipartite graphs, unpublished manuscript (1982)

3. Chang, G.J., Nembauser, G.L.: "The $k$-domination and $k$-stability problem of graphs", Report No. 540, School of OR\&IE, Cornell University, Ithace, N.Y. (1982)

4. Damaschke, P., Müller, H., Kratsch, D.: Domination in convex and chordal bipartite graphs. Inf. Process. Lett. 36, 231-236 (1990)

5. Erdös, P.: Problems and Results in Combinatorial Analysis; Proceedings, Eighth Southeastern Conference on Combinatorics, Graph Theory and Computing, Congressus Numerantium XIX (F. Hoffman et al. Eds.) 3-12, (1977)

6. Lemke, Paul: A Counterexample to a Conjecture of Abbott. J. Comb. Theory, Ser. A 50, 301-304 (1989)

Received: May 20, 1993

Revised: October 21, 1994 